\title{
Excess low-frequency noise in AIGaN/GaN-based high-electron-mobility transistors
}

\author{
S. A. Vitusevich, ${ }^{a), b)}$ S. V. Danylyuk, a) and N. Klein \\ Institut für Schichten und Grenzflächen, Forschungszentrum-Jülich, Jülich D-52425, Germany \\ M. V. Petrychuk, V. N. Sokolov, V. A. Kochelap, and A. E. Belyaev \\ Institute of Semiconductor Physics, NASU, Kiev 03028, Ukraine
}

V. Tilak, J. Smart, A. Vertiatchikh, and L. F. Eastman

School of Electrical Engineering, Cornell University, Ithaca, New York 14853

(Received 27 December 2001; accepted for publication 28 January 2002)

\begin{abstract}
The low-frequency $1 / f$ noise characteristics of $\mathrm{AlGaN} / \mathrm{GaN}$ high-electron-mobility transistors with gate length scaled down to $150 \mathrm{~nm}$ grown on sapphire by metalorganic chemical vapor deposition have been studied. Certain features of the $1 / f$ noise have been revealed in these short-gate transistors. The low-frequency noise spectra show drastically different behavior depending on the gate voltage $V_{G}$ in the range of low $\left(V_{G t} \leqslant V_{G} \leqslant 0\right)$ and high $\left(V_{G}<V_{G t}\right)$ biases. The noise spectra-gate bias dependences allow one to distinguish a spatial redistribution of effective noise sources in the transistor channel. The Hooge parameter has been deduced separately for the ungated region, $\alpha_{H}^{p} \simeq 10^{-3}$, and for the gated region, $\alpha_{H}^{a} \simeq 2 \times 10^{-4}$, of the transistor channel. These values are as low as those previously observed in nitride heterostructures grown on silicon carbide substrates. (ㄷ) 2002 American Institute of Physics. [DOI: 10.1063/1.1463202]
\end{abstract}

The group III-nitride-based electronic devices are very attractive for power applications at high frequencies. Nitride material systems offer great potential for providing much higher output power, operational voltage, and temperature. Due to a unique combination of high current density, high breakdown electric field, and good thermal conductivity, these materials are in the focus of state-of-the-art semiconductor research. ${ }^{1-3}$ The low-frequency $1 / f$ noise is one of the important factors that limits the device performance and determines its capability for power microwave applications. The level of such noise and corresponding value of the Hooge parameter may often be significant in $\mathrm{AlGaN} / \mathrm{GaN}$ structures depending on growth technique and different type of substrates (mainly sapphire and silicon carbide are used as most suitable). In previous studies, a low-frequency noise was measured in high-electron-mobility transistor (HEMT) devices with gate length of a micrometer range scale. ${ }^{4-11}$ The devices with a shorter gate length have smaller characteristic transit times and provide higher operating frequencies. Moreover, certain features of carrier transport and noise can manifest themselves with scaling down the device feature lengths, which will impact the device performance. In this letter, we present the study of low-frequency noise of AlGaN/GaN-based HEMTs with gate length scaled down to deep submicrometer range of $150 \mathrm{~nm}$.

$\mathrm{AlGaN} / \mathrm{GaN}$ HEMTs under investigation were grown by metalorganic chemical vapor deposition on sapphire substrates. A $40 \mathrm{~nm} \mathrm{AlGaN} \mathrm{(16 \%} \mathrm{Al)} \mathrm{nucleation} \mathrm{layer} \mathrm{grown} \mathrm{on}$ the substrate was followed by the deposition of a $1.1 \mu \mathrm{m}$ nominally undoped GaN buffer layer and a $23 \mathrm{~nm} n$-AlGaN

\footnotetext{
${ }^{a}$ On leave from the Institute of Semiconductor Physics of National Academy of Science of the Ukraine, 03028, Kiev, Ukraine.

${ }^{b)}$ Electronic mail: s.vitusevich@fz-juelich.de
}

(33\% Al) undoped barrier layer. Transistors were fabricated using Ti/Al/Ti/Au metallization annealed for $40 \mathrm{~s}$ at $800{ }^{\circ} \mathrm{C}$ for source and drain ohmic contacts and $\mathrm{Ni} / \mathrm{Au}$ for Schottky gate contacts. The surface was covered with $320 \mathrm{~nm} \mathrm{Si}_{3} \mathrm{~N}_{4}$. The devices had gate length of $0.15,0.25,0.30,0.35 \mu \mathrm{m}$, gate width of 100-400 $\mu \mathrm{m}$, and drain-gate spacing of $1 \mu \mathrm{m}$. A source-drain spacing was of $3 \mu \mathrm{m}$. A complete direct current characterization has been carried out in HEMT devices with variety of channel widths and lengths. Hall mobility and sheet carrier concentration in ungated structures were extracted from Hall measurements. A room temperature electron mobility of $1250 \mathrm{~cm}^{2} / \mathrm{V} \mathrm{s}$ at a sheet carrier density of $1.05 \times 10^{13} \mathrm{~cm}^{-2}$ was measured in the channel.

The transistor dc characteristics were investigated with a HP 4145B semiconductor parameter analyzer operated in transistor mode. Low-frequency noise measurements were performed at different gate-source voltages in the common source configuration under open circuit conditions $R_{L} \gg r_{d}$, where $R_{L}$ is the load resistance in the drain circuit and $r_{d}$ is the channel differential resistance. The noise spectra were measured in the frequency range from $1 \mathrm{~Hz}$ to $100 \mathrm{kHz}$ at $300 \mathrm{~K}$ with using low-noise preamplifier ITHACO 1201. The directly measured noise power density $S_{V m}(f)$ was analyzed by HP35670 A Dynamic Signal Analyzer. Then, the noise spectra of the transistors were obtained by $S_{V}(f)$ $=\left[S_{V m}(f) / G^{2}-S_{V_{b}}(f)\right] / k(f)$, where $S_{V_{b}}(f)$ is the background noise of the low-noise preamplifier, $G$ and $k(f)$ are the gain of the preamplifier and a correction factor to a nonuniformity of the gain $G$ frequency behavior (normalized amplitude-frequency characteristic), respectively. We used a set of wire load resistors $R_{L}$ which did not contribute an additional excess noise. The observed peculiarities in $1 / f$ noise spectra have been exhibited most distinctly in shortgate devices. Next, we concentrate on results obtained for the 


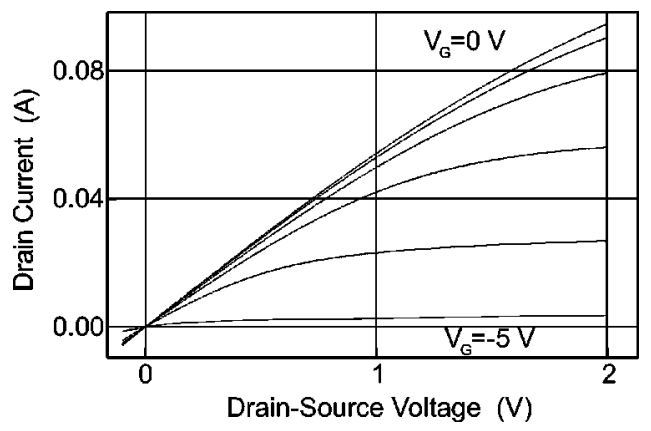

FIG. 1. The dc $I-V$ characteristics of the transistor measured for different gate voltage $V_{G}$ from 0 to $-5 \mathrm{~V}$ with step $\Delta V_{G}=-1 \mathrm{~V}$.

device with $0.15 \mu \mathrm{m}$ gate length and $400 \mu \mathrm{m}$ gate width.

The set of the transistor dc current-voltage $(I-V)$ characteristics at different gate biases is presented in Fig. 1. It is seen that the $I-V$ curves can be divided into two groups which are discriminated by a threshold value of the gate bias $V_{G t}=-3 \mathrm{~V}$, for $V_{G} \geqslant V_{G t}$ and for $V_{G}<V_{G t}$. The former, corresponding to the low gate voltage measurements, is characterized by very weak deviation from linear behavior (even at a relatively high drain-source voltage of $V_{D} \approx 2 \mathrm{~V}$ ) and a weak dependence on the gate bias. On the contrary, the latter corresponds to the transistor operation practically in subsaturation regime at such gate biases, so that only initial part of the $I-V$ curves $\left(V_{D} \lesssim 150 \mathrm{mV}\right)$ is linear. Thereby, $V_{G}=V_{G t}$ is the threshold gate bias which distinguishes different regimes of the transistor operation with weak and strong control of the channel resistance.

The voltage-noise spectral density $S_{V}(f)$ was obtained for different gate voltages in the range of $0--5 \mathrm{~V}$ and for drain currents in the range of 1.03-25.9 mA. The spectra show frequency dependence of the type of $1 / f^{\gamma}$ with the exponent $\gamma$ being very close to unity ( $1 / f$ noise). The background noise of preamplifier became apparent only for relatively small noise levels at the very high frequencies used in the experiment, which will be beyond our considerations. The gate voltage of about $-3 \mathrm{~V}$ has been found to discriminate the noise spectra behavior for both the frequency dependence and the drain-source current dependence at a given frequency of the analysis. In Fig. 2, we show two groups of the normalized spectra of the drain-source voltage noise at different gate biases, $V_{G}>-3 \mathrm{~V}$ (curves 1 and 2) and $V_{G}$

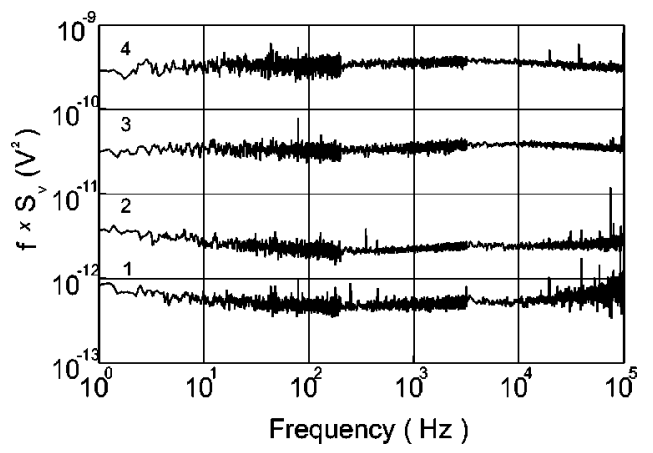

FIG. 2. Normalized frequency spectra of the voltage noise at certain gate biases below (curves 1 and 2) and above (curves 3 and 4) the threshold voltage $V_{G t}: 1-V_{G}=0 \mathrm{~V}, I_{D}=5.65 \mathrm{~mA}, V_{D}=111 \mathrm{mV} ; 2-V_{G}=0 \mathrm{~V}, I_{D}$ $=15.4 \mathrm{~mA}, V_{D}=303 \mathrm{mV} ; 3-V_{G}=-4.5 \mathrm{~V}, I_{D}=1.24 \mathrm{~mA}, V_{D}=92 \mathrm{mV}$

$4-V_{G}=-4.5 \mathrm{~V}, I_{D}=11.7 \mathrm{~mA}$, and $V_{D}=635 \mathrm{mV}$. Redistribution subject to AIP license or copyright, see http://apl.aip.org/apl/copyright.jsp
Downloaded $21 \mathrm{Dec} 2006$ to 134.94 .122 .39 . Redis

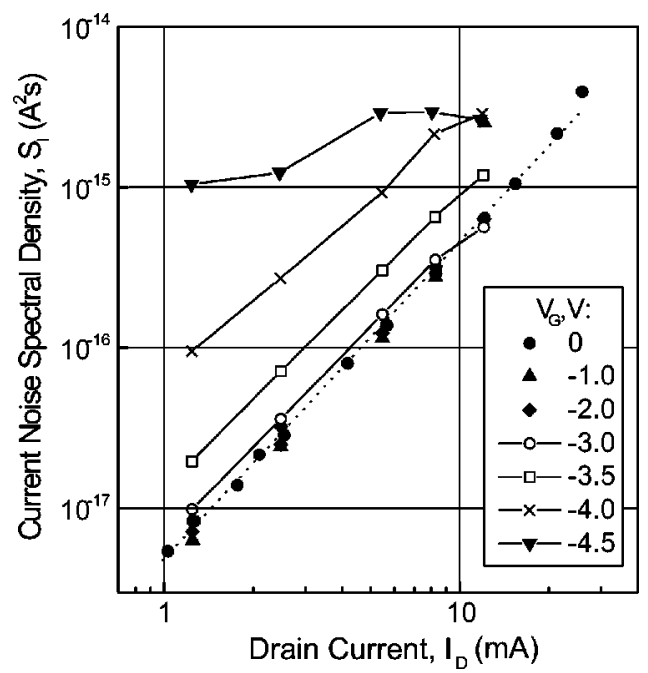

FIG. 3. Dependences of current-noise spectral density on the drain current $I_{D}$ measured at frequency $f=10 \mathrm{~Hz}$ and different gate biases $V_{G}$ from 0 to $-4.5 \mathrm{~V}$; the dotted curve shows the quadratic dependence of $S_{I}$ vs $I_{D}$.

$<-3 \mathrm{~V}$ (curves 3 and 4 ). Within each group of curves, the gate voltage is the same whereas the drain current is different. The spectra shape does not depend on the drain current, while it is changed with the gate bias. For the first group of measurements, an increase in the noise spectral power $S_{V}(f)$ is observed (with respect to the $1 / f$ dependence) at the very low frequencies, as well as somewhat elevated values of high-frequency contributions. In the spectra of the second group, the low-frequency growth is not observed and there is a maximum at frequencies of $3-10 \mathrm{kHz}$ dependent on the source-drain voltage. These peculiarities in the noise spectra may be due to the occurrence of local levels with distinct characteristic time constants which need further investigation.

For the analysis of main contributions to the observed noise, we study the dependences of the drain current-noise spectral density $S_{I}(f)=S_{V}(f) / r_{d}^{2}$ on the drain current $I_{D}$ for different gate biases and for a given frequency $f=10 \mathrm{~Hz}$ (Fig. 3). It is remarkable that the same value of the gate bias, $V_{G t}$, obtained for the dc $I-V$ characteristics, determines in Fig. 3 two regions where the noise level is weakly and strongly controlled with the gate voltage. The studies of the origin of the low-frequency noise in field-effect transistors are performed usually in the linear regime of operation. We analyze the results of the noise measurements relevant to this regime [ $-4.5 \mathrm{~V} \leqslant V_{G} \leqslant 0 \mathrm{~V}$ and $V_{D} \lesssim 150 \mathrm{mV}$ (Fig. 1)], considering the two groups of curves distinguished in Figs. 1 and 3 .

Concerning the first group of measurements $(-3 \mathrm{~V}$ $\leqslant V_{G} \leqslant 0 \mathrm{~V}$ ), it is to be noted that differential resistance of the channel is varied only weakly, $r_{d} \approx 20 \Omega$ for $V_{D}$ $\leqslant 150 \mathrm{mV}$ (Fig. 1), and the noise level practically is not changed with the gate voltage (Fig. 3). The dependence of $S_{I}$ on $I_{D}$ is strictly quadratic. This proves that the measured noise is not a contact one but caused by fluctuations of the channel resistance. It is usually characterized by the dimensionless Hooge parameter, $\alpha_{H},{ }^{12}$

$$
\alpha_{H}=\frac{S_{I}(f)}{I_{D}^{2}} f N,
$$


where $N$ is the total number of the conduction electrons in the noise region. With simple manipulations, we get from Eq. (1) the expression

$$
\alpha_{H}=\frac{S_{I}(f)}{V_{D}^{2}}\left(f L^{2} R / q \mu_{n}\right),
$$

where $L$ is the channel length, $R=L^{2} /\left(q \mu_{n} N\right)$ is the channel (ohmic) resistance, $\mu_{n}$ is the electron mobility, and $q$ is the electron charge. Using the data of Figs. 1 and 3, the Hooge parameter calculated by Eq. (2) at $V_{G}=0, V_{D}=100 \mathrm{mV}, I_{D}$ $=5 \mathrm{~mA}$, and $L=0.15 \mu \mathrm{m}$ is as low as $\alpha_{H}=2.7 \times 10^{-6}$, which is an incredibly small quantity. Such a discrepancy can be eliminated if we take that the device noise for the considered range of gate biases (in the linear regime of operation) is determined by the noise sources located in passive (ungated) regions adjacent to the drain and source contacts. In this regime, the resistance of the active (gated) region is a small part of the total resistance of the transistor channel between the drain and source contacts. Indeed, if we use in Eq. (2) the distance $L=3 \mu \mathrm{m}$, then we obtain $\alpha_{H}=10^{-3}$. This value of $\alpha_{H}$ is comparable to the standard magnitude ${ }^{12}$ of $\alpha_{H}=2 \times 10^{-3}$, as well as to that previously obtained for nitride heterostructures grown on sapphire substrates. ${ }^{5,6,8}$

The second group of curves in Fig. $3\left(V_{G}<-3 \mathrm{~V}\right)$ is characterized by a lower slope of the dependence $S_{I}\left(I_{D}\right)$, with the slope being decreased with increasing the gate bias. Moreover, the noise spectral level $S_{I}$ for this group of measurements exceeds the respective values of $S_{I}$ for the first group of curves discussed herein.

Such a distinct separation in the behavior of the spectral noise density $S_{I}\left(I_{D}\right)$ at $V_{G}=-3 \mathrm{~V}$ allows one to distinguish between noise coming from passive $\left(\alpha_{H}=\alpha_{H}^{p}\right)$ and active $\left(\alpha_{H}=\alpha_{H}^{a}\right)$ regions of the transistor channel. Assuming uncorrelated noise contributions from different regions, the voltage noise of active region, $S_{V}^{a}$, can be determined by subtracting the noise of passive region, $S_{V}^{p}$, from the total noise power $S_{V}=S_{V}^{p}+S_{V}^{a}$, that has been measured in the experiment under given dc current regime. The dc and noise performance of the passive region can be determined from experimental results corresponding to zero gate bias, i.e., we take the resistance of the passive region to be $R_{p}$ $=\left.\left(V_{D} / I_{D}\right)\right|_{V_{G}=0}$ and the respective voltage-noise spectral density to be $S_{V}^{p}=S_{V}\left(V_{G}=0\right)$. Respectively, for the active region we can write $S_{I}^{a}=S_{V}^{a} /\left(r_{d}^{a}\right)^{2}$, where differential resistance of the active region is just its ohmic resistance $r_{d}^{a}$ $=V_{D}^{a} / I_{D}$ (linear regime), and $V_{D}^{a}=V_{D}-I_{D} R_{p}$ is the voltage drop across the active region. Thus, the Hooge parameter attributed to the active region of the transistor channel can be calculated by

$$
\alpha_{H}^{a}\left(V_{G}\right)=\frac{S_{I}^{a}\left(f, V_{G}\right)}{\left(V_{D}^{a}\right)^{2}}\left(f L_{a}^{2} R_{a} / q \mu_{n}\right) .
$$

Here, we have assumed the electron mobility to be the same all over the passive and active regions. From Eq. (3), the value of the Hooge parameter has been extracted, $\alpha_{H}^{a}\left(V_{G}\right)$ $\cong 2 \times 10^{-4}$. It is found for the investigated transistors that $\alpha_{H}^{a}$ is independent on the gate bias in the range of $-4.5 \mathrm{~V}$ $\leqslant V_{G} \leqslant-3 \mathrm{~V}$.

In conclusion, the $1 / f$ noise of $\mathrm{AlGaN} / \mathrm{GaN}$ HEMTs grown on sapphire with the gate length scaled down to nanometer range has been studied. Contributions to the noise from different regions of the transistor channel has been revealed. A Hooge parameter has been extracted separately for passive (ungated) and active (gated) regions of the channel being $\alpha_{H}^{p} \cong 10^{-3}$ and $\alpha_{H}^{a} \cong 2 \times 10^{-4}$, respectively. These prove a good noise quality of the active region of the transistor channel (comparable with that for the devices grown on $\mathrm{SiC}$ ). We suggest that optimization of the transistor design via reduction of the passive regions will considerably improve the $1 / f$-noise figures of nanoscale-gate-length AlGaN/ GaN HEMTs grown on sapphire substrates.

This work is supported by the Office of Naval Research, Grant No. N00014-01-1-0828 (Project Monitor Dr. Colin Wood). Two of the authors (V.N.S. and V.A.K.) acknowledge support by the State Foundation for Fundamental Research of Ukraine, and one of the authors, (A.E.B.) is grateful to the Deutsche Forschungsgemeinshaft for a research grant.

${ }^{1}$ O. Ambacher, J. Phys. D 31, 2653 (1998).

${ }^{2}$ S. J. Pearton, F. Ren, A. P. Zhang, and K. P. Lee, Mater. Sci. Eng., R. 30, 55 (2000).

${ }^{3}$ S. J. Pearton, F. Ren, A. P. Zhang, G. Dang, X. A. Cao, K. P. Lee, H. Cho, B. P. Gila, J. W. Johnson, C. Monier, C. R. Abernathy, J. Han, A. G. Baca, J.-I. Chyi, C.-M. Lee, T.-E. Nee, C.-C. Chuo, and C. N. G. Chu, Mater. Sci. Eng., B 82, 227 (2001).

${ }^{4}$ S. Rumyantsev, M. E. Levinshtein, R. Gaska, M. S. Shur, A. Khan, J. W. Yang, G. Simin, A. Ping, and T. Adesida, Phys. Status Solidi A 176, 201 (1999).

${ }^{5}$ J. A. Garrido, F. Calle, E. Muñoz, I. Izpura, J. L. Sanchez-Rojas, R. Li, and K. L. Wang, Electron. Lett. 34, 2357 (1998).

${ }^{6}$ A. Balandin, S. V. Morozov, S. Cai, R. Li, K. L. Wang, G. Wijeratne, and C. R. Viswanathan, IEEE Trans. Microwave Theory Tech. 47, 1413 (1999).

${ }^{7}$ N. Pala, R. Gaska, S. Rumyantsev, M. S. Shur, M. A. Khan, X. Hu, G. Simin, and J. Yang, Electron. Lett. 36, 268 (2000).

${ }^{8}$ S. Rumyantsev, M. E. Levinshtein, R. Gaska, M. S. Shur, J. W. Yang, and M. A. Khan, J. Appl. Phys. 87, 1849 (2000).

${ }^{9}$ A. Balandin, K. L. Wang, S. Cai, R. Li, C. R. Viswanathan, E. N. Wang, and M. Wojtowicz, J. Electron. Mater. 29, 297 (2000).

${ }^{10}$ J. A. Garrido, B. E. Foutz, J. A. Smart, J. R. Shealy, M. J. Murphy, W. J. Schaff, L. F. Eastman, and E. Muñoz, Appl. Phys. Lett. 76, 3442 (2000).

${ }^{11}$ S. L. Rumyantsev, N. Pala, M. S. Shur, E. Borovitskaya, A. P. Dmitriev, M. E. Levinshtein, R. Gaska, M. A. Khan, J. Yang, X. Hu, and G. Simin, IEEE Trans. Electron Devices 48, 530 (2001).

${ }^{12}$ F. N. Hooge, T. G. M. Kleinpenning, and L. K. J. Vandamme, Rep. Prog. Phys. 44, 479 (1981). 